$5-30-2020$

\title{
Quand et comment la loi est-elle efficace pour réduire la pratique des MGF/E: une étude transfrontalière au Burkina Faso et au Mali
}

Josephine Wouango

Susan L. Ostermann

Daniel Mwanga

Population Council

Follow this and additional works at: https://knowledgecommons.popcouncil.org/departments_sbsr-rh

Part of the International Public Health Commons, and the Social and Behavioral Sciences Commons How does access to this work benefit you? Let us know!

\section{Recommended Citation}

Wouango, Josephine, Susan L. Ostermann, and Daniel Mwanga. 2020. "Quand et comment la loi est-elle efficace pour réduire la pratique des MGF/E: une étude transfrontalière au Burkina Faso et au Mali," Evidence to End FGM/C Policy Brief. Nairobi: Population Council. 


\section{Quand et comment la loi est-elle efficace pour réduire la pratique des $M G F / E$ : une étude transfrontalière au Burkina Faso et au Mali}

\section{Le consortium du programme Evidence to End FGM/C produit des données probantes pour informer et influencer les investissements, les politiques et les programmes visant à mettre fin aux mutilations génitales \\ féminines/excision dans différents contextes. \\ Population Council Lead Institution \\ Africa Coordinating Centre for the Abandonment of Female Genital \\ Mutilation/Cutting, Kenya (ACCAF)}

Global Research and Advocacy Group, Senegal (GRAG)

MannionDaniels Ltd. (MD)

Population Reference Bureau (PRB)

University of Washington (Prof. Bettina Shell-Duncan)

University of California, San Diego (Dr. Gerry Mackie)

\section{Funded by}

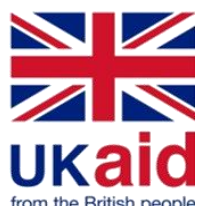

\section{Introduction}

Les mutilations génitales

féminines/excision (MGF/E) sont reconnues dans le monde entier comme étant associées à des risques pour la santé physique et psychologique des filles et des femmes. Elles sont également considérées comme une violation des droits humains. À I'heure actuelle, on estime que 200 millions de filles et de femmes dans 30 pays ont subi une MGF/E et que 30 millions de filles de moins de 15 ans sont à risque (UNICEF 2016). Une réponse politique commune aux MGF/E consiste à promulguer des lois et à faire respecter les interdictions pénales.

Au Burkina Faso et au Mali, les types de MGF/E pratiquées sont en grande partie les types I et II de l'OMS. En 1996, le Burkina Faso a adopté une loi punissant la pratique. Cette loi a été révisée en 2018 avec une évolution vers des peines d'emprisonnement et des amendes supplémentaires. Au Mali, le gouvernement a mis en place une stratégie pour mettre fin à la pratique, mais, à ce jour, il n'a pas adopté de loi qui criminalise spécifiquement les MGF/E. Au Burkina Faso, les statistiques nationales suggèrent qu'il y a eu une baisse de la prévalence chez les femmes âgées de 15 à 49 ans ( $76 \%$ en 2010 à $67 \%$ en 2015) et chez les filles âgées de 0 à 14 ans (13\% en 2010 à $11 \%$ en 2015) (INSD 2012, 2015). Au Mali, la prévalence de la pratique serait plus élevée, avec environ $91 \%$ des femmes âgées de 15 à 49 ans qui ont subi une excision en 2012-13 (INFO-Stat/Mali et ICF International 2014).
Cette note de synthèse résume les principales conclusions d'une étude transfrontalière portant sur deux pays voisins : le Burkina Faso, qui dispose d'une loi forte sur les MGF/E, et le Mali, qui n'a pas de loi spécifique sur les MGF/E (Wouango, Ostermann, et Mwanga 2020). Les objectifs de l'étude transfrontalière étaient d'explorer et de comparer les attitudes et les tendances en matière de respect de la loi et de poursuite de la pratique des MGF/E, et de comprendre l'efficacité du droit pénal dans la lutte contre les MGF/E dans les deux pays.

\section{Méthodes}

Cette étude a utilisé une méthodologie mixte (quantitative et qualitative). Les données ont été collectées dans six villages jumelés le long de la frontière entre le Burkina Faso et le Mali, un endroit où la prévalence des MGF/E est élevée parmi les groupes ethniques dominants. Chaque village jumelé a été choisi de manière à refléter le même groupe ethnique majoritaire (les Bobo à Faramana-BF/Koury-Mali, les Senoufo à Koloko-BF/Finkolo-Mali et les Bwaba à Tansila-BF/Boura-Mali). Notre population cible était composée d'hommes et de femmes âgés de plus de 18 ans. Notre étude quantitative a été menée auprès d'un échantillon aléatoire de participants $(n=1209)$ et comprenait une méthode innovante du list experiment technique pour évaluer l'intention future des répondants de 
pratiquer les MGF/E. Cette technique permet aux chercheurs de poser des questions sur des sujets sensibles ou sur des activités socialement inacceptables tout en dissimulant les réponses de l'enquêté(e) au chercheur et à toute autre partie. Elle aide ainsi à surmonter le biais de la désirabilité sociale souvent associé aux enquêtes basées sur des déclarations,

Le volet qualitatif de l'étude comprenait des entretiens avec des informateurs clés (EIC) $(n=60)$ et des discussions de groupe (DG) $(n=24)$. Les informateurs clés ont été sélectionnés à dessein en fonction de leurs positions au sein des communautés choisies et comprenaient des chefs communautaires et religieux, des représentants d'ONG locales et des fonctionnaires tels que des agents de santé, des préfets, des enseignants et des travailleurs sociaux. Tous les informateurs clés étaient âgés de 18 ans ou plus. Les DG ont été menées auprès d'hommes et de femmes d'âges divers répartis en quatre groupes : hommes plus jeunes (18-34 ans), hommes plus âgés (35 ans et plus), femmes plus jeunes (18-34 ans) et femmes plus âgées ( 35 ans et plus). Chaque groupe était composé de 6 à 8 personnes. Les participants étaient tous membres de la communauté et ont été recrutés par les chefs d'équipe qualitatifs avec le soutien des facilitateurs locaux.

L'approbation éthique a été obtenue auprès du ministère de la Santé du Mali, du ministère de la Santé du Burkina Faso et du comité d'éthique du Population Council.

\section{Résultats}

\section{Que savent les participants sur la légalité ou l'illégalité des MGF/E?}

La grande majorité des personnes interrogées pensent que la pratique des MGF/E est illégale ( 87 $\%$ de tous les répondants au Burkina Faso et $80 \%$ au Mali). Les résultats du Burkina Faso sont conformes aux études précédentes (CNLPE 2006). Les résultats du Mali sont surprenants, dans la mesure où le Mali ne dispose pas d'une loi spécifique qui criminalise et punit la pratique des MGF/E. La radio a été citée comme la principale source d'information sur la loi dans les deux pays pour la majorité des participants à l'étude. Les informations sur les MGF/E à la radio sont transmises par les fonctionnaires du gouvernement tels que les agents de santé, les juges et les travailleurs sociaux, ainsi que par les ONG locales et les dirigeants locaux. Au Burkina Faso, ces acteurs jouent un rôle clé dans le soutien des actions du Comité national de lutte contre la pratique de l'excision (CNLPE). Les messages visent principalement à sensibiliser le public en expliquant les conséquences des MGF/E sur la santé, en informant sur la loi et ses sanctions, et sur les possibilités de signaler les cas d'excision (ou intentions) de manière anonyme grâce à la ligne téléphonique gratuite "SOS excision ». Par exemple, les médias couvrent toutes les audiences foraines publiques, ce qui rend le processus bien connu de la population, même dans les régions éloignées. De plus, le CNPLE utilise la radio pour expliquer les possibilités de réparation des séquelles de l'excision et des fistules obstétricales. La radio locale est le média le plus accessible aux populations de nos zones d'étude, car elle émet dans la langue locale commune, le dioula. Le fait que les participants citent la radio comme principale source d'information sur la loi au Mali peut s'expliquer en partie par une diffusion transfrontalière de messages anti-MGF/E en provenance du Burkina Faso.

« [...] Il a été dit à la télévision et à la radio par des médecins que les filles ne devaient pas subir d'excision. »- Homme, EIC, Koury, Mali

« II y a des émissions de radio où le juge vient même expliquer au public certaines dispositions de la loi. » - Femme, EIC, Tansila, Burkina Faso

\section{Qu'est-ce qui motive les gens à obéir ou à désobéir à une loi interdisant les MGF/E?}

Le fait qu'il faut respecter la loi a motivé un peu plus de la moitié des répondants à déclarer qu'ils obéissaient eux-mêmes à la loi: $54 \%$ ont déclaré que la motivation à obéir à la loi découle d'un sentiment d'obligation légale et de la conviction que les autres se comportent de la même manière (54,4 \% au Burkina Faso et 53,6 \% au Mali). En outre, $84 \%$ des personnes interrogées au Burkina Faso et $77 \%$ au Mali ont déclaré qu'elles ne poursuivraient pas les MGF/E si tous les autres membres de leur communauté abandonnaient cette pratique. Cela renforce l'importance des normes sociales dans la détermination du comportement en matière de MGF/E, comme le soulignent deux répondants de l'EIC ci-dessous :

Q : Qu'arriverait-il à une famille qui déciderait de ne pas exciser sa fille?

« Eh bien, ce sera un isolement social. Puisque vous appartenez à une communauté qui a ses lois, et lorsque vous contestez ces lois, c'est comme si vous vous mettiez hors de la communauté. Donc, c'est surtout cet isolement, votre façon de voir et de faire les 
choses qui vous met à l'écart de la communauté. Vous avez refusé de suivre les règles communautaires et c'est ce qui peut vous mettre à l'écart. »-EIC, Finkolo, Mali

«Rien ne leur arrivera dans notre communauté. Aujourd'hui, il y a beaucoup de ménages où les filles de moins de 20 ans ne sont pas excisées. II y a tant de filles qui sont dans cette situation aujourd'hui, donc rien ne leur arrivera. » - EIC, Koloko, Burkina Faso

\section{Comment la loi sur les MGF/E du Burkina Faso a-t-elle été mise en œuvre?}

La loi a été appliquée par le biais de différentes approches. Les tribunaux mobiles organisent des poursuites et des condamnations publiques au sein des communautés, les cas (ou intentions) d'excision peuvent être signalés par le biais d'une ligne téléphonique "SOS Excision », et il existe une forte collaboration entre les parties prenantes, une volonté politique, une communication efficace et une sensibilisation dans les langues locales. Cette approche à multiples facettes est possible grâce à la forte implication des forces de sécurité et des chefs religieux et locaux. Le CNLPE au Burkina Faso a entrepris une stratégie appelée « approche par les leaders communautaires » qui consiste à engager les leaders communautaires et religieux respectés à adhérer au message d'abandon et à le transmettre. Les enregistrements de leur engagement et de leurs messages sont diffusés à la radio, à la télévision, dans les journaux et sur les pages web des médias sociaux du CNLPE. Par exemple, le Moro Naba de Ouagadougou, qui est le roi suprême des Mossis au Burkina Faso, est un champion du CNLPE pour l'abandon des MGF/E. Les messages portent sur les conséquences sanitaires de cette pratique et les agents de santé sont formés et impliqués en tant qu'acteurs clés dans des zones très éloignées. Toutefois, les interventions portant spécifiquement sur les MGF/E transfrontalières font défaut. II n'y a pas d'intervention coordonnée forte entre les deux pays.

Les personnes interrogées ont également fréquemment mentionné les agents de santé comme la source de leurs connaissances sur la loi interdisant les MGF/E (au Burkina Faso), ainsi que sur les nombreuses conséquences négatives de la pratique sur la santé (dans les deux pays).
«Nous ne le faisons plus parce que les médecins nous ont dit de ne pas le faire. "-Homme, EIC, Faramana, Burkina Faso

« J'ai appris avec les agents de santé qui nous ont dit de ne plus le faire parce que la loi interdit d'exciser les filles. »-Femme, GD 35+,

Faramana, Burkina Faso

"Les médecins [agents de santé] ont déclaré à la radio et à la télévision que nous ne devrions plus exciser les filles. »- Homme, GD 35+, Koury, Mali

«Les agents de santé nous ont dit que l'excision des filles n'est pas une bonne chose et qu'il fallait y mettre fin. Ils arrêtent même des gens pour cela. Aujourd'hui, certaines personnes le font en secret, pour d'autres, le mari est d'accord, pour d'autres encore, le mari n'est pas d'accord. »- Femme, GD 18-34, Boura, Mali

\section{Quels sont les facteurs qui entravent l'abandon des MGF/E?}

Les participants ont fourni diverses raisons pour justifier la poursuite de la pratique : culture/tradition, des raisons sociales (possibilité de se marier), les avantages perçus en matière de santé, la pression des anciens, la régulation du désir sexuel des femmes, les sanctions sociales telles que l'humiliation, et les conséquences au sein de la communauté et de son réseau de référence. Le respect de la culture et de la religion, en particulier, a un effet significatif sur le comportement des gens : certains répondants pensent que la pratique est autorisée par leur religion (15,8\% au Burkina Faso et $18,3 \%$ au Mali) et leurs coutumes $(20,7 \%$ au Burkina Faso et $22,5 \%$ au Mali).

Les MGF/E sont pratiquées à travers la frontière du Burkina Faso et du Mali (Wouango, Ostermann, and Mwaya 2020) ainsi qu'à travers les frontières avec la Côte d'Ivoire, le Niger et le Ghana (GRIGED 2008), ce qui entrave son abandon.

" Je sais que c'est lors des mariages que l'on dit souvent que cette fille n'est pas excisée. II y a même des filles qui ont été renvoyées chez leurs parents parce qu'elles n'ont pas été excisées.

Cela signifie que pour beaucoup de parents, c'est la même raison qui les a poussés à exciser leurs filles. Ils ne veulent pas qu'un jour son mari la répudie parce qu'elle n'est pas excisée. »Homme, EIC, Koloko, Burkina Faso 


\section{Recommandations}

Le gouvernement du Burkina Faso devrait poursuivre ses approches innovantes actuelles d'application de la loi anti-MGF/E, qui incluent : des audiences foraines qui sensibilisent le public tout en condamnant publiquement les exciseuses et leurs complices; une ligne téléphonique gratuite permettant de signaler les cas d'excision, gérée par les forces de sécurité mises à la disposition du SP/CNLPE; une stratégie efficace de lobbying et de plaidoyer par l'engagement de leaders communautaires et religieux de haut niveau; des formations des acteurs judiciaires, des agents de sécurité, des agents de santé; des patrouilles

communautaires effectuées par les forces de sécurité pour sensibiliser les membres de la communauté; des partenariats étroits avec les médias nationaux et locaux qui couvrent toutes les audiences foraines et diffusent largement les activités et les messages nationaux de lutte contre les MGF/E en français et dans les langues locales; la traduction de la loi dans les quatre principales langues nationales et sa diffusion dans les communautés par l'intermédiaire d'organisations locales; l'engagement d'anciennes exciseuses par des déclarations publiques d'abandon des MGF/E; et le rôle prépondérant du CNLPE dont la mission est de coordonner toutes les actions en partenariat avec de nombreux acteurs et qui doit être soutenu par des ressources conséquentes.

Des recommandations supplémentaires spécifiques sont :

- Continuer à impliquer les agents de santé, les travailleurs sociaux et à les soutenir par des ressources et des activités de renforcement des capacités. Ce sont des agents de changement qui ont eu une influence positive sur les femmes de nos zones d'étude.

- Mettre en œuvre des programmes interpayset renforcer la coopération pour éliminer les MGF/E transfrontalières. Nos résultats ont révélé que la coopération régionale est nécessaire pour réduire la pratique transfrontalière des MGF/E. À ce jour, il n'existe pas de mécanisme formel qui aborde cette question. Une loi régionale qui intensifie les efforts au niveau communautaire au-delà de la frontière devrait contribuer à réduire la pratique souterraine de transfrontalière.

- Le gouvernement du Mali devrait adopter et appliquer, à l'instar des autres pays de la sous-région, une loi spécifique interdisant les MGF/E et pourrait s'inspirer de l'expérience du Burkina Faso. II devrait élaborer des stratégies pour persuader les chefs religieux et communautaires puissants et influents d'encourager l'abandon parmi leurs fidèles, et fournir des ressources et un soutien aux agents de santé qui semblent jouer un rôle clé dans le passage à l'abandon.

\section{Références}

\section{GRIGED. 2008. Pratique transfrontalière de} l'excision : État des lieux et évaluation des actions dans les zones frontalières $d u$ Burkina Faso, de la Côte d'Ivoire, du Ghana, du Mali et du Niger. Study Report. New York: UNIFEM.

INFO-Stat/Mali and ICF International. 2014. Enquête démographique et de santé au Mali 2012-2013. Rockville MD: CPS, INSTAT, INFO-STATE, and ICF International.

Institut National de la Statistique et de la Démographie (INSD), Ministère de l'Économie et des Finances, Burkina Faso. 2012. Enquête démographique et de santé et à indicateurs multiples (EDSBF-MICS IV) 2010. Calverton, MD: ICF International.

\section{. 2015. Enquête multisectorielle continue} (EMC). Burkina Faso: INSD

National Committee to Fight the Practice of Excision (CNLPE). 2016. Evaluation de l'impact des activités de promotion de l'élimination de la pratique de l'excision de 1990 à 2015 au Burkina Faso. Final Report. Ouagadougou: Ministry of Family, National Solidarity and Women.

UNICEF. 2016. Female Genital Mutilation/Cutting: A Global Concern. New York: UNICEF.

Wouango, J., S. Ostermann, and D. Mwanga. 2020, "When and How Is the Law Effective in Reducing the Practice of FGM/C. A CrossBorder Study in Burkina Faso and Mali," Evidence to End FGM/C: Research to Help Girls and Women Thrive. New York: Population Council. 